Meta

Journal des traducteurs

Translators' Journal

\title{
Comment traduire « to affect » ?
}

\section{Jean Delisle}

Volume 21, numéro 4, décembre 1976

URI : https://id.erudit.org/iderudit/002525ar

DOI : https://doi.org/10.7202/002525ar

Aller au sommaire du numéro

Éditeur(s)

Les Presses de l'Université de Montréal

ISSN

0026-0452 (imprimé)

1492-1421 (numérique)

Découvrir la revue

Citer cet article

Delisle, J. (1976). Comment traduire « to affect » ? Meta, 21(4), 274-277.

https://doi.org/10.7202/002525ar

Ce document est protégé par la loi sur le droit d'auteur. L'utilisation des services d'Érudit (y compris la reproduction) est assujettie à sa politique d'utilisation que vous pouvez consulter en ligne.

https://apropos.erudit.org/fr/usagers/politique-dutilisation/
Cet article est diffusé et préservé par Érudit.

Érudit est un consortium interuniversitaire sans but lucratif composé de l’Université de Montréal, l'Université Laval et l'Université du Québec à Montréal. Il a pour mission la promotion et la valorisation de la recherche. https://www.erudit.org/fr/ 


\section{COMMENT TRADUIRE «TO AFFECT»?}

Le verbe TO AFFECT est omniprésent en anglais. On le retrouve aussi bien dans des écrits à caractère général que dans des descriptions médicales, des textes législatifs ou des rapports technico-scientifiques. Le traducteur novice est porté à le rendre invariablement par son homonyme français "affecter ". Dans certains contextes, cependant, cette solution de facilité énerve le sens et prive le texte de langue d'arrivée soit de nuances utiles à sa bonne intelligence soit de termes colorés qui en rendraient la lecture plus agréable. Savoir rendre les finesses de l'original avec élégance fait aussi partie de l'art du traducteur.

La mort d'un parent peut m'affecter, mais puis-je vraiment dire qu'une usine hydroélectrique " affecte " le milieu naturel? Que les riverains d'un aéroport " sont affectés " par le bruit des aéronefs? Qu'une mesure d'expropriation "affecte " le droit de propriété des citoyens? Cet article vise à préciser les cas où il y a 
concordance parfaite entre TO AFFECT et AFFECTER, et à proposer des équivalents plus justes aux nombreux emplois abusifs de ce verbe français qui, sous l'influence de l'anglais, est en voie de devenir au Canada français un mot passepartout. Dans sa façon d'appréhender la réalité, le français manifeste une prédilection pour les contours nets et une certaine répugnance à l'égard des formes nébuleuses.

La lexicologie différentielle nous a appris à procéder au recoupement de l'aire sémantique des unités lexicales pour délimiter les zones de chevauchement sémantique. En français, l'aire sémantique du verbe AFFECTER se compose de nombreuses acceptions. Il y a lieu de distinguer celles qui sont issues du verbe latin AFFECTARE (rechercher avec ardeur) et celles qui découlent de AFFICERE (toucher, émouvoir).

A. AFFECTER (étym. : affectare)

1. Adopter une attitude qui manque de sincérité ou de naturel. (Elle affecta un air méprisant.)

2. Désigner, nommer une personne à un poste. (Affecter un fonctionnaire à tel ministère.)

3. Destiner à un usage précis. (Crédits affectés à la construction.)

4. Aimer beaucoup, employer avec prédilection. (Affecter certaines expression.)

5. S'efforcer de paraître ce que l'on n'est pas en réalité. (Il affecte de paraître savant.)

6. (Au figuré) Revêtir une forme. (Les gouttes affectent une forme ronde.)

7. (Au figuré) Sens moral. (L'ambition affecte des formes différentes.)

B. AFFECTER (étym. : afficere)

1. Agir sur (en bien ou en mal) ${ }^{1}$. (Ce médicament affecte les poumons.)

2. (Droit) Frapper de charges financières. (Domaine affecté d'hypothèques. Mortgaged - Dans ce sens, on dit plutôt grevé.)

3. (Algèbre) Modifier une quantité en la dotant d'un signe. (Quantité affectée d'un exposant. - Bearing an index.)

4. (Au figuré) Subir une impression. (Son échec l'a vivement affecté.)

Telles sont les acceptions modernes les plus courantes de ces deux homographes. En anglais, l'aire sémantique du verbe TO AFFECT est tout aussi étendue. Dans l'usage courant, ce verbe évoque en outre "l'idée d'une influence

1. Le comité de linguistique de Radio-Canada précise que le verbe AFFECTER s'emploie le plus souvent en mauvaise part dans le sens médical ou moral. (Fiche AFFECTER $n^{\circ} 293$ ) 
exercée d'une façon quelconque ${ }^{2}$ "sur quelqu'un ou sur quelque chose. C'est cet emploi assez vague que le Webster rend par " to act upon ... to produce a material influence upon (Rainfall affects plant growth) or alteration in (Areas to be affected by highway). "Dans ce sens, TO AFFECT exprime une modification qui souvent n'est pas un changement en profondeur. La question est de savoir si en français AFFECTER peut'signifier altérer. Pouvons-nous dire par exemple que " le calcium "affecte " la carrosserie des voitures"? Ou encore que "les changements qui se produisent sur l'île d'Orléans "affectent " l'image familière de cette île "? Les ouvrages sur les difficultés de la langue française ne sont pas tout à fait muets sur le sujet ${ }^{3}$. S'appuyant sur la définition de l'Académie, l'auteur du Dictionnaire des difficultés grammaticales et lexicologiques estime qu'on peut dire au figuré "Cette discussion a affecté notre amitié " et qu'il est normal de donner à ce verbe le sens figuré d'altérer. Au sens propre, il convient cependant de le remplacer par un verbe plus précis pour exprimer l'altération. On dira donc plus justement "le calcium RONGE / CORRODE / ATTAQUE la carrosserie des voitures ". Et dans la seconde phrase " affectent " sera remplacé avantageusement par des verbes tels que MODIFIER / ALTÉRER / TRANSFORMER.

En outre, l'emploi du verbe AFFECTER au sens de viser, concerner, intéresser, toucher est un anglicisme. On dira donc que telle décision CONCERNE les employés, que les propriétaires sont VISÉS par telle loi, ou encore que telle mesure PORTE ATTEINTE à la liberté d'expression. C'est évidemment le contexte qui permet de préciser le sens à donner au verbe TO AFFECT qui véhicule souvent un aspect négatif qu'on peut rendre en français par des verbes ou des locutions verbales tels que NUIRE À, GÊNER, ÊTRE PRÉJUDICIABLE À, FAIRE TORT À, LÉSER, etc. La destination du texte, sa fonction, oblige parfois à employer des locutions neutres telles que AVOIR UN EFFET, UNE INFLUENCE, UNE INCIDENCE SUR / INFLUER SUR, pour rendre le sens "d'influence exercée sur "véhiculé par TO AFFECT.

D'autre part, le traducteur fait preuve de "créativité d'expression " chaque fois qu'il remplace par un verbe imagé le verbe "affecter "dans son sens abusif de modifier. Par exemple, la phrase "des développements désordonnés ont "affecté " la beauté des paysages et la noblesse des vieux établissements" devient beaucoup plus claire, plus élégante et plus expressive si son unique verbe est remplacé par deux "verbes-métaphores". La phrase transformée pourrait se lire ainsi : " des développements désordonnés ont TERNI la beauté des paysages et AVILI la noblesse des vieux établissements. " La traduction y gagne toujours. Il y aurait d'ailleurs beaucoup à dire sur la liberté rédactionnelle dont jouit le traducteur.

2. Pierre Daviault, Langage et traduction, Ottawa, Information Canada, 1962. (Réimpression 1972). L'auteur propose de nombreux équivalents, certains en contexte, pour ce verbe de haute fréquence.

3. Joseph Hanse, Dictionnaire des difficultés grammaticales et lexicologiques, Paris, Les éditions scientifiques et littéraires, 1971 , p. 72 . Voir aussi l'article que Dupré a consacré au mot AFFECTER dans son Encyclopédie du bon français dans l'usage contemporain, Paris, les Editions Trévise, 1972. 
En guise de conclusion, une dizaine d'exemples suffiront à démontrer qu'il est facile et souvent préférable de puiser dans les ressources du français pour trouver une solution plus élégante et plus juste que le calque systématique pour traduire le verbe TO AFFECT.

Precipitation was above normal in areas affected by the storm.

Les précipitations ont été supérieures à la normale dans les régions BALAYÉES par la tempête.

These problems may affect the safe operation of the vehicle.

Ces défauts peuvent NUIRE à la sécurité đu véhicule.

Euphony is so powerful that it has affected syntactic patterns.

"L'euphonie est si forte qu'elle a su INFLÉCHIR les structures syntaxiques ${ }^{4}$."

The increase expenditures has had an adverse impact on the balance of payments and development programs are likely to be affected in many countries.

Cet accroissement des dépenses a eu une incidence défavorable sur la balance des paiements et les programmes de développement risquent d'être COMPROMIS dans plusieurs pays.

Each factor affects all the other, and when the decisions are made, they must be re-examined from time to time.

Tous les facteurs sont INTERDÉPENDANTS, et, même lorsque les décisions sont arrêtées, une révision s'impose de temps à autre.

Persons who now live around Malton will not be affected by the noise of the air traffic.

Les riverains de Malton ne seront pas INCOMMODÉS par le trafic aérien.

The energy crisis that strikes the U.S.A. will affect Canada very soon.

Le Canada RESSENTIRA prochainement LES CONTRECOUPS de la crise de l'énergie qui sévit aux États-Unis.

Canadian economic activity is affected by seasonal unemployment.

L'activité économique canadienne n'ÉCHAPPE pas au chômage saisonnier.

Our way of life has been profoundly affected by electricity.

L'électricité a BOULEVERSÉ notre mode de vie.

Alcohol will affect your driving ability.

(Traduction relevée dans une brochure de la Société des alcools du Québec : " L'alcool affecte vos capacités dans la conduite d'un véhicule. "Il faut être ivre-mort pour pondre pareille horreur!)

L'alcool RÉDUIT votre aptitude à conduire (un véhicule).

L'alcool AFFAIBLIT vos facultés au volant.

Jean Delisle

4. Irène de Buisseret, Deux langues, six idiomes, Ottawa, Carlton-Green, 1975, p 88. 